\title{
ON THE ORBITAL PERIOD DISTRIBUTION OF RECYCLED PULSARS
}

\author{
L. A. NELSON ${ }^{1}$, S. RAPPAPORT ${ }^{2}$ \\ 1. Physics Dept., Bishop's Univ., Lennoxville, QC, Canada \\ 2. CSR, Room 37-551, MIT, Cambridge, MA 02139, USA
}

\begin{abstract}
Using population synthesis techniques, we investigate the orbital period distribution of 'recycled' pulsars. We find that the relative distribution of systems with periods between approximately one and fifty days are reasonably well reproduced. However, the existence of binary pulsars with periods between 70 and $\sim 1000 \mathrm{~d}$ cannot be explained within the evolutionary framework considered here.
\end{abstract}

\section{Introduction}

More than 50 recycled radio pulsars have been discovered. Of the pulsars known to be members of binary systems, most are believed to contain low-mass degenerate companions in nearly circular orbits having orbital periods in the range of several hours to $1000 \mathrm{~d}$. According to one evolutionary scenario, an evolved star initially undergoes stable mass transfer onto a neutron star companion and leaves behind a degenerate core [3]. During the process, the neutron star is 'spun up'. In our calculations, we explicitly follow the evolution of these systems from the moment when Roche lobe overflow occurs until the cessation of mass transfer. The initial conditions were determined by using population synthesis techniques. In all cases, the neutron star was assumed to have a mass of $1.4 \mathrm{M}_{\odot}$. Details of the evolutionary calculations will be presented elsewhere [1].

\section{Conclusions}

If the secondary (donor) is sufficiently evolved at the onset of mass transfer, nuclear evolution of the secondary dominates the evolution of the binary. As a result, the orbital period generally increases until the mass in the envelope of the (sub)giant is exhausted. The end product is a detached binary consisting of a 'recycled' pulsar and a degenerate dwarf companion. Most of these systems seem to obey a well defined $P_{\text {orb }}-M_{\text {wd }}$ relationship

\section{5}

A. Evans and J. H. Wood (eds.), Cataclysmic Variables and Related Objects, 475-476.

(C) 1996 Kluwer Academic Publishers. Printed in the Netherlands. 


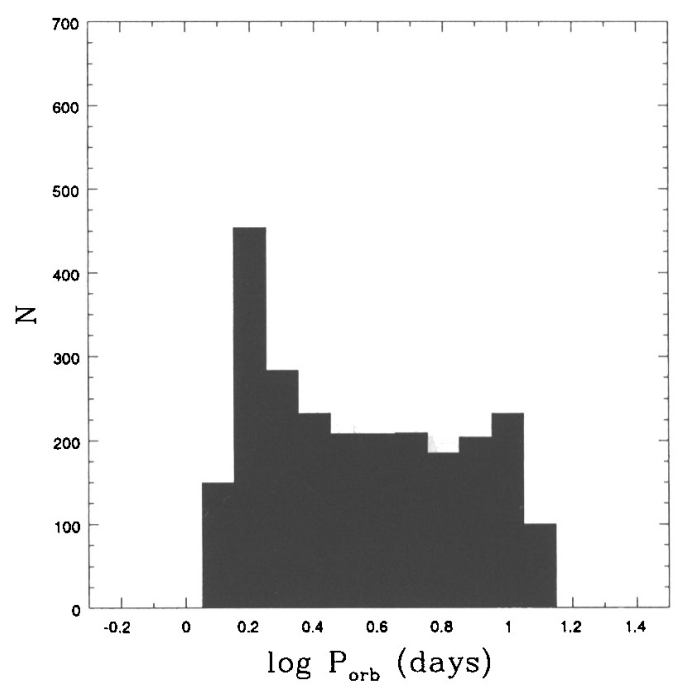

Figure 1. Relative orbital period distribution of simulated binary pulsars containing low-mass degenerate companions in the Galactic disk at the current epoch. The original neutron star progenitor was chosen to have a mass between 10 and $30 \mathrm{M}_{\odot}$ and the mass of the secondary was taken to be uncorrelated with respect to the primary's mass (a fit to the Miller \& Scalo IMF was used). The efficiency $(\alpha)$ of the removal of the envelope during the common envelope phase was taken to be unity. Largely unevolved secondaries (i.e. those that did not develop a significant helium core) and systems suffering dynamical instability were removed from the population.

(the exceptions being systems with small $M_{\mathrm{wd}}$ ). We find that most of the binary pulsar systems that are formed have orbital periods between 1 and $15 \mathrm{~d}$ (see Fig. 1). This accounts for the paucity of systems in the 'gap' of the orbital period distribution between 15 and $70 \mathrm{~d}$. The apparent decrease in the number of observed systems with periods of less than $1 \mathrm{~d}$ can be understood in terms of the bifurcation [2] of the theoretical tracks. The existence of binary pulsars with periods between 70 and $\sim 1000 \mathrm{~d}$ cannot be explained within the evolutionary framework considered here. This problem requires further investigation [1].

\section{References}

1. Nelson, L. A., Rappaport, S., 1996, in preparation

2. Pylyser, E. H. P., Savonije, G. J., 1988, A\&A, 191, 57

3. Rappaport, S., Podsiadlowski, P., Joss, P. C., et al., 1995, MNRAS, 273, 731 